EPJ Web of Conferences 62, 07004 (2013)

DOI: $10.1051 /$ epjconf/20136207004

(C) Owned by the authors, published by EDP Sciences, 2013

\title{
Fission dynamics: The quest of a temperature dependent nuclear viscosity
}

\author{
E. Vardaci ${ }^{1, a}$, A. Di Nitto ${ }^{2}$, P.N. Nadtochy ${ }^{3}$, A. Brondi ${ }^{1}$, G. La Rana ${ }^{1}$, R. Moro ${ }^{1}$, M. Cinausero ${ }^{4}$, \\ G. Prete ${ }^{4}$, N. Gelli ${ }^{5}$, E.M. Kozulin ${ }^{6}$, and G.N. Knyazheva ${ }^{6}$ \\ ${ }^{1}$ Istituto Nazionale di Fisica Nucleare and Diparimento di Fisica, Universitá degli Studi di Napoli \\ “Federico II', Napoli 80126, Italy \\ ${ }^{2}$ Johannes Gutenberg- Universität Mainz Institut für Kernchemie, Mainz, Germany \\ ${ }^{3}$ Omsk State University, Mira prospekt 55-A, Omsk 644077, Russia \\ ${ }^{4}$ Laboratori Nazionali di Legnaro dell' Istituto Nazionale di Fisica Nucleare, Legnaro (Padova) 35020, \\ Italy \\ ${ }^{5}$ Istituto Nazionale di Fisica Nucleare, Sesto Fiorentino (Firenze) 50019, Italy \\ ${ }^{6}$ Flerov Laboratory of Nuclear Reactions, Joint Institute for Nuclear Research, RU-141980 Dubna, \\ Moscow Region, Russia
}

\begin{abstract}
This contribution presents a journey within the open questions about the current use of a temperature dependent nuclear viscosity in models of nuclear fission and proposes an alternative experimental approach by using systems of intermediate fissility. This study is particularly relevant because: i) systems of intermediate fissility offer a suitable framework since the intervals between the compound nucleus and scission point temperatures with increasing excitation energy are much smaller than in the case of heavier systems, ii) the measurement of observables in the ER channel translates into a larger set of effective constraints for the models.
\end{abstract}

\section{Introduction}

A large variety of studies [1-10] on the fission decay of composite systems with $\mathrm{A} \approx 180-250$ has shown that the pre-scission multiplicities of neutrons and charged particles increase monotonically with the bombarding energy in contrast with the calculations of the statistical model (SM). This finding is considered as the evidence that fission is a slow process with respect to the lifetime for the emission of light particles. With increasing excitation energy, the particle decay lifetime decreases and becomes smaller than the time necessary for the build up of the collective motion of the nuclear matter toward the saddle point. Consequently, fission does not compete as effectively as predicted by the SM in the early stages of the decay, and particles and $\gamma$-ray emissions can occur more favorably. In other words, there is a delay in the collective motion toward the fission barrier not accounted for in the statistical picture. The

\footnotetext{
${ }^{a}$ e-mail: vardaci@na.infn.it
}

This is an Open Access article distributed under the terms of the Creative Commons Attribution License 2.0, which permits unrestricted use, distribution, and reproduction in any medium, provided the original work is properly cited. 
overall cause of the establishment of these transient effects is believed to be associated with the nuclear matter viscosity which slows down the collective flow of mass from equilibrium to scission. A strong dissipation due to nuclear viscosity can indeed trigger a variety of effects of dynamical origin, among which that a compound nucleus committed to fission (already at the saddle point configuration) can still became an evaporation residue if enough particles are evaporated and the fissility reduced [5].

Most of the estimates of the fission time scale have been obtained from the neutron prescission multiplicities on the basis of the SM [1]. However, several variants of the SM have been proposed in the literature to take explicitly into account transient effects, time scales as well as viscosity $[1,2,6,7]$. Following the initial idea of the "neutron clock" [1], the common trend is to split the path from the equilibrium to the scission point configuration into two regions, the pre- and the post-saddle. The total fission time is defined as $\tau_{f}=\tau_{d}+\tau_{s s c}$, where $\tau_{d}$ is the pre-saddle delay, namely, the characteristic time which the composite system spends inside the barrier, and $\tau_{s s c}$ is the time necessary to travel the path from saddle to scission. The relevant observables are computed using $\tau_{d}$ and $\tau_{s s c}$ as free parameters, along with the other input parameters relative to the specific ingredients of the model, and fit to the experimental data. However, $\tau_{d}$ and $\tau_{s s c}$ are considered dependent on another parameter, the viscosity parameter $\gamma$, as well. Following Kramer's work [11], the inclusion of dissipative effects results in an effective time-dependent fission decay width $\Gamma_{f}(t)$ which is smaller than the standard Bohr-Wheeler decay width by a hindrance factor,

$$
\Gamma_{f}(t)=\Gamma_{B W}\left[\sqrt{1+\gamma_{d}^{2}}-\gamma_{d}\right]\left[1-\exp \left(-\tau / \tau_{d}\right)\right] .
$$

Here $\tau_{d}$ is a delay parameter, $\Gamma_{B W}$ is the Bohr-Wheeler fission decay width, and $\gamma_{d}$ is the nuclear viscosity parameter in the pre-saddle region which can be written as $\gamma_{d}=\beta / 2 \omega_{o} . \beta$ is the so-called reduced dissipation parameter and $\omega_{o}$ is the potential curvature at the saddle point. Also the saddle to scission time $\tau_{s s c}$, might be dependent on the nuclear viscosity parameter $\gamma_{s s c}$ in the post-saddle region. One ansatz widely used is the following:

$$
\tau_{s s c}=\tau_{s s c}(\gamma=0)\left[\sqrt{1+\gamma_{s s c}^{2}}+\gamma_{s s c}\right] .
$$

In general, the nuclear viscosity parameter might be different inside and outside the saddle point. Furthermore, $\tau_{d}, \tau_{s s c}, \gamma_{d}$ and $\gamma_{s s c}$ are dependent on the available excitation energy, the temperature of the nucleus, the fission barrier, the angular momentum.

In spite of the extensive work, estimates of the fission time scales are however quite controversial, ranging from 5 to $500 \times 10^{-21} \mathrm{~s}$, depending on the system and on the experimental probe. Furthermore, such estimates are weakened by the fact that different sets of input parameters can result in equally good fits within the same model $[3,4,7]$.

Dynamical models [12-18], based on the Euler-Lagrange, Fokker-Planck or Langevin equations, have been used in order to estimate the reduced viscosity parameter $\beta$ and to gain insight on the nature of dissipation. A set of collective degrees of freedom is chosen and the internal degrees of freedom constitute the heath bath. Dissipation is the mechanism set up to transfer energy between collective and internal degrees of freedom. The evolution of the collective variables on a potential energy surface describes the fission process.

One of the main issues is whether the nuclear dissipation mechanism proceeds primarily by means of individual two-body collisions (two-body friction), as in the case of ordinary fluid, or by means of nucleons colliding with a moving potential wall (one-body friction). The analysis of the fission fragment TKE [12], using the one-body or two-body prescriptions in the dissipation function, indicates that this observable is not sufficient alone to elucidate this point. Two-dimension Langevin equation has been used to analyze the TKE and the prescission neutron multiplicity for the nucleus ${ }^{200} \mathrm{~Pb}$ [13]. In this case, one-body dissipation allows reproducing both quantities, while unusually strong two-body viscosity 
allows reproducing only neutron multiplicity. Similarly, the values of the reduced viscosity parameter $\beta=6 \times 10^{21} s^{-1}, 15 \times 10^{21} s^{-1}$ and $24 \times 10^{21} s^{-1}$, extracted from the prescission neutron multiplicities for the composite nucleus ${ }^{188} \mathrm{Pt}$ at $\mathrm{Ex}=66.3$, 99.7 and 101.4 $\mathrm{MeV}$ [19], are consistent with one body dissipation, and indicate that $\beta$ should increase with temperature.

A different result was found for the system ${ }^{220}$ Th by Rubchenya et al. [6] by applying a revised SM: the effective average value of $\beta$ decreases with increasing excitation energy, similarly to the temperature dependence expected for the two-body friction. This result is in striking contrast with the result of Hofman et al. [3] where their set of data is equally well reproduced by a friction coefficient $\gamma$ which increases with either $\mathrm{T}_{\text {saddle }}$ or $\mathrm{T}_{\text {saddle }}^{2}\left(\mathrm{~T}_{\text {saddle }}\right.$ is the temperature at the saddle point), and, in any case, increases with the projectile energy.

In conclusion, the estimates of $\beta$, both from statistical and dynamical models, provide a contradictory picture on the values of $\beta$, which range from $\approx 2$ to $30 \times 10^{21} s^{-1}$, and result in rather controversial conclusions on the nature of nuclear dissipation and its dependence on the shape and temperature.

\section{Dynamical vs. statistical approach}

There are few characteristics of the description of the fission process that come out of these two deeply different approaches that are quite surprising. In the SM, the viscosity parameters are treated as a constant free parameters to be adjusted on the experimental data. It turns out that the viscosity is higher in the post-saddle path than in the pre-saddle one and increases with the temperature or the square of the temperature. Light particles and/or GDR $\gamma-$ rays are emitted mostly in the post-saddle region where viscosity is higher. Added to this is the fact that the same data can be reproduced equally well if the parameter $\gamma$ is taken to be temperature or deformation dependent $[7,8]$.

In the dynamical approach the compound nucleus can pass the saddle point several times before eventually undergoing fission and no free parameter is possible in the dissipation model (one- or twobody) except for a strength parameter $[17,18]$. Contrarily to what occurs in the statistical approach, viscosity is higher in the pre-saddle and, hence, light particles and/or GDR $\gamma$ - rays are emitted mostly in the pre-saddle region. In both one-body or two-body dissipation there is no dependence on the temperature.

The question is: who is right and how can we disentangle this apparent contradiction. Somehow the answer could be simple because the statistical approach can only mimic a dissipation model by introducing $a d-h o c$ parameters. However, it must be pointed out that only neutron multiplicities and GDR $\gamma$ - rays have been measured in most of the studies and mostly for heavy systems $(\mathrm{A} \geq 200)$, and the lack of a sufficient number of constraints to the models could, in several cases, be the source of discrepancies. In order to withdraw a more consistent picture of nuclear dissipation, and its connection with the shape and the temperature, it seems crucial the take into account a larger number of observables which can be expected to be sensitive to the nuclear dissipation and to try to reproduce the variety of observables with a unique set of input parameters.

\section{Dissipation in systems of intermediate fissility}

The systems of intermediate fissility $(\chi=0.5-0.6)$ offer quite a unique environment where nuclear viscosity can be studied [20]. They are characterized by an evaporation residue (ER) cross-section comparable or larger than the fission cross-section, and by a shorter path in the deformation space from the saddle-to-scission point [21]. Consequently: 1) the input parameters of the models can be further constrained by the energy spectra and multiplicities of the light particles in the ER channel; 2) the effect of the fission delay over the fission and ER cross section is much more pronounced with respect to heavier systems because the emission of a charged particle in the pre-saddle region strongly enhances the probability of producing an evaporation residue as consequence of both a reduction of the fissility 

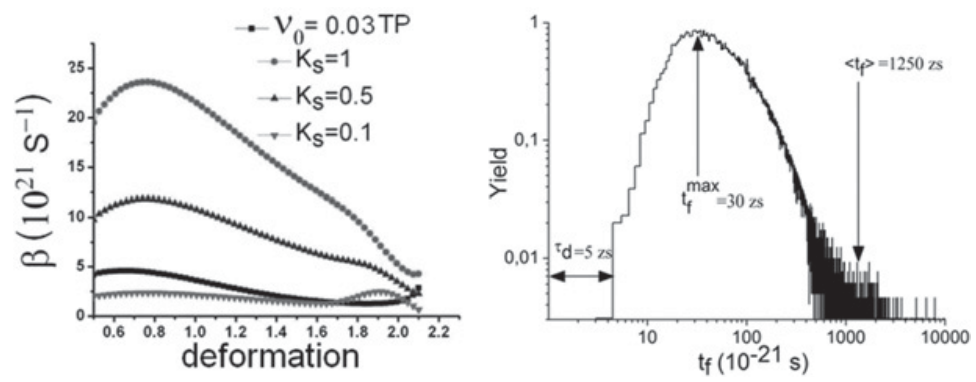

Figure 1. Left: reduced viscosity parameter vs. deformation of the compound nucleus. $\mathrm{K}_{s}$ is the strength of the one-body dissipation. Full strength is for $\mathrm{K}_{s}=1$. The black solid line is the functional dependency expected in the case of the two-body. Right: calculated distribution of the fission times in the system ${ }^{32} \mathrm{~S}+{ }^{100} \mathrm{Mo}$ at $200 \mathrm{MeV}$.

and the large value of the angular momentum necessary to ignite fission. The fact that the potential energy surface is characterized by a shorter path from the saddle to scission means that the role of the pre-saddle dynamics relative to the saddle to scission dynamics is enhanced and, hence, some of the ambiguities on the not-well identified separation and interplay between pre- and post-saddle might be reduced in the interpretation of the data. We expect that the measurements of neutron and charged particle multiplicities and energy spectra in the two channels as well as the measurements of the cross sections of the channels themselves will allow to put more severe constraints onto the models. This should provide more reliable values of fission delay and of the viscosity parameter, and contribute to a better comprehension of the nuclear viscosity.

In the system ${ }^{32} \mathrm{~S}+{ }^{100} \mathrm{Mo}$ at $200 \mathrm{MeV}$ of bombarding energy we have measured at LNL with the $8 \pi$ LP setup several quantities which are expected to be affected by nuclear dissipation, namely, TKEMass distribution of the fission fragments, the fission and ER cross sections, protons and alpha particle multiplicities in both the ER and fission channels. In a first step, this whole set of data was analyzed in the frame of the SM [22-26] and it was concluded that although the SM is able to reproduce the observables related to the fission channel alone without delay, it overestimates the multiplicities of the protons and alpha particles in the ER channel. The only way found to reproduce the whole set of data is through the use of a dynamical model in which the fission process, along with the evaporation of light particles in the fission and ER paths, is treated with a 3D-Langevin approach coupled to the SM [25]. In particular, only the full one-body dissipation mechanism, with viscosity dependent on the shape of the fissioning nucleus and not on the temperature, is able to reproduce satisfactorily the whole set of data.

In Figure 1 (left) we show how the reduced friction coefficient varies with the deformation of the nucleus en route toward fission in the one-body dissipation model. The case that is able to give the best agreement with the full set of data is represented by $\mathrm{K}_{s}=1$, namely, full one-body dissipation ("wall" and "wall-plus-window") [25]. The two-body dissipation case is represented by the full thick line. It is clear that one-body dissipation shows a stronger dependence on the deformation. Furthermore, the viscosity grows at the beginning of the deformation until a maximum is reached; afterwards, it decreases monotonically for increasing deformation. This means that viscosity shows the maximum strength only at the beginning of the collective motion and when the shape is still fairly compact. No dependence on the temperature is assumed so far. In Figure 1 (right) the time distribution of all fission events shows that fission can take place in quite a large interval of time. What is normally used in the statistical approach does not correspond to any of the characteristic times of the distribution above and this confirms the inadequacy of the SM approach to describe nuclear dissipation. The extension of the time distribution may also explain why different time scales are extracted with the SM approach when different probes are used [25]. 

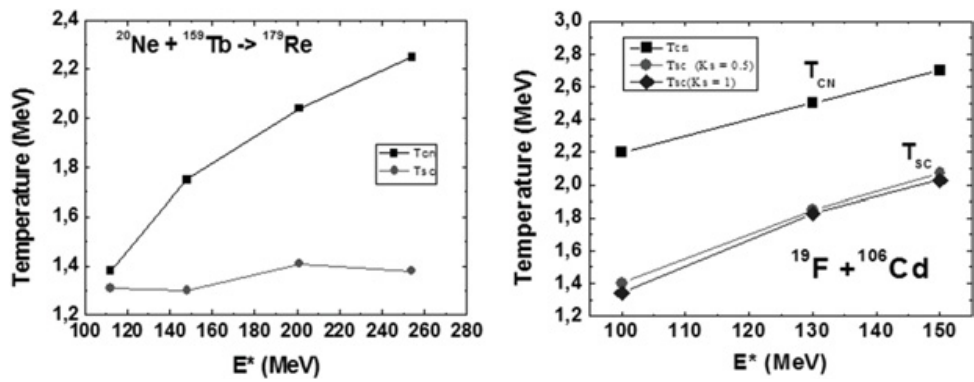

Figure 2. Left: pre- and post-scission temperatures vs. initial excitation energy of the compound nucleus [10]. Right: pre- and post-scission temperatures vs. initial excitation energy of the compound nucleus in the case of an intermediate mass system calculated with a dynamical model [17]. $\mathrm{K}_{s}=1$ is for full one-body dissipation.

\section{How to probe a temperature-dependent viscosity}

The nuclear mechanism of dissipation may indeed depend on the temperature. In many experimental studies on heavy systems it is commonly found that if the temperature of the compound nucleus $\mathrm{T}_{C N}$ is raised, the temperature at scission point $T_{S C}$ remains almost constant $[1,10,27]$. This supports the picture in which fission occurs after that the composite nucleus has cooled down until has reached an excitation energy of roughly $50-60 \mathrm{MeV}$, independently on the excitation energy of the initial compound nucleus. This is shown in Figure 2 (left) [10].

If a study on the dependence of nuclear viscosity on the temperature is performed with this picture in mind (for instance by measuring the excitation function of some observables of the kind above), inevitably the measurable effects on the observables, which are deduced from the deviations from the model used, are averaged over an interval of temperatures $\Delta \mathrm{T}=\mathrm{T}_{C N}-\mathrm{T}_{S C}$ which grows with the bombarding energy. In other words, as the interval of temperatures $\mathrm{T}_{C N}-\mathrm{T}_{S C}$ increases, the observable effects are integrated over a larger and larger interval of temperatures, and a possible dependence on the temperature is hidden. Consequently, heavy systems are not the ideal frame for the study of the dependence of the viscosity parameter from the temperature.

Systems of intermediate fissility have instead a completely different behavior. With our model $[17,18]$ we calculated $\mathrm{T}_{S C}$ for several systems. In Figure 2 (right) we show the dependence of $\mathrm{T}_{C N}$ and $\mathrm{T}_{S C}$ from the initial excitation energy of the compound nucleus for the case of the system ${ }^{19} \mathrm{~F}+{ }^{106} \mathrm{Cd}$. The interesting feature of these systems is that $\mathrm{T}_{S C}$ also grows with the excitation energy of the initial compound nucleus, a behavior at variance with that found for heavier systems. This is due to the delicate balance between the time scale of the fission process and the binding energy of the pre-scission particles: the neutron prescission emission, which is mainly responsible for the cooling in the pre-saddle region, is suppressed by the higher binding energy and stronger competition with charged particles. As a result, the two fission fragments retain most of the initial excitation energy.

\section{Conclusions and perspectives}

The analysis presented here remarks how promising is the dynamical approach in describing both fission and ER channel within the same model. A dynamical model allows to penetrate more intimate details of the fission process. At the same time, the model can be more and more refined if more observables are measured for the same system. Of course, the model should be oriented on the calculations of quantities that are directly linked to measured observables. In this respect, we have enlarged the computational capabilities of our code to include the calculation of energy spectra and angular distribution of the prescission particles. This is a novel feature that constraints even more the model parameters and in turn 
allows to disentangle more characteristic features of the fission process. Furthermore, in this contribution we have also proposed an alternative scenario where the study of the dependence of the nuclear viscosity from the temperature seems more suitable.

The reported study was partially supported by RFBR, research project No. 13-02-00168

\section{References}

[1] D. Hilscher and H. Rossner, Ann. Phys. (France) 17, 471 (1992), and references therein

[2] J. P. Lestone et al., Nucl. Phys. A 559, 277 (1993)

[3] D. J. Hofman et al, Phys. Rev. C 51, 2597 (1995)

[4] A. Chatterijee et al, Phys. Rev. C 52, 3167 (1995)

[5] L. Fiore et al., Nucl. Phys. A 620, 71 (1997)

[6] V. A. Rubchenya et. al, Phys. Rev. C 58, 1587 (1998)

[7] I. Dioszegi et al., Phys. Rev. C 61, 024613 (2000)

[8] B. B. Back et al. Phys. Rev. C 60, 044602 (1999)

[9] G. La Rana et al., Eur. Phys. J. A 16, 199 (2003)

[10] J. Cabrera et. al, Phys. Rev. C 68, 34613 (2003)

[11] A. Kramer, Physica (Amsterdam) 7, 284 (1940)

[12] A. J. Sierk et al., Phys. Rev. C 21, 982 (1980)

[13] T. Wada et al., Phys. Rev. Lett. 70, 3538 (1993)

[14] A. V. Karpov et al., Phys. Rev. C 63, 54610 (2001)

[15] G. Chaudhuri and S. Pal, Phys. Rev. C 65, 54612 (2002)

[16] P. Frobrich and I. I. Gontchar, Europhys. Lett. 57, 355 (2002)

[17] P. N. Nadtochy et al., Phys. Rev. C 65, 64615 (2002)

[18] P. N. Nadtochy et al., Phys. Lett. B 685, 258 (2010)

[19] C. Badimon, Ph.D. Thesis.- CENBG, Bordeaux, (2001)

[20] E. Vardaci et al., Dynamics Aspects of Nuclear Fission, Casta-Papiernicka, Slovak Republic, Oct 1998, eds. Yu. Ts. Oganessian, J. Kliman, S. Gmuca (World Scientific Co., 2000) p. 261

[21] M.G. Itkis, A. Ya. Rusanov, Phys. Part. Nucl. 29, 160 (1998)

[22] E. Vardaci et al, AIP Conf. Proc. 1175, 57 (2009)

[23] A. Di Nitto et al., AIP Conf.Proc. 1175, 346 (2009)

[24] E. Vardaci et al., Eur. Phys. J. A 43, 127 (2010)

[25] P. N. Nadtochy et al., EPJ Web Conf. 2, 8003 (2010)

[26] A. Di Nitto et al., Eur. Phys. J. A 47, 83 (2011)

[27] E. Mordhorst et al., Phys. Rev. C 43, 716 (1991) 